\title{
Manifestaciones hemorrágicas en el dengue.
}

\section{Artículo Original}

Ramón Rivas-Llamas ${ }^{1}$, Ricardo A. Chaín-Castro ${ }^{2}$, Joel Murillo-Llanes ${ }^{3}$, María G. Ramírez-Zepeda ${ }^{3}$ Tania Gadea-Alvarez², Esperanza López-López ${ }^{1}$.

${ }^{1}$ Depto. de Hematología, Banco de Sangre y Unidad de Aféresis, ${ }^{2}$ Depto. de Medicina Interna, ${ }^{3}$ Depto. de Investigación, Hospital General de Culiacán SSA “Dr. Bernardo J. Gastelum”, Culiacán, Sinaloa, México.

\section{RESUMEN.}

Objetivo.- Describir las manifestaciones clínicas y epidemiológicas en pacientes con fiebre por dengue (FD) y fiebre hemorrágica por dengue (FHD). Material y Métodos.- Estudio transversal de 231 casos de dengue atendidos en el Hospital General de Culiacán "Dr. Bernardo J. Gastelum", de septiembre a noviembre de 2003, clasificados según los criterios de la OMS. Se utilizó el programa estadístico Stata v6. Resultados.- 209 (90.48\%) fueron FD y 22 (9.52\%) FHD. La edad promedio fue de $34.5 \pm 15$ años. La FD predominó en mujeres, con 129 casos $(61.7 \%)$ y la FHD en hombres con 17 casos (77.3\%). En la FHD acudieron al hospital en $4.7 \pm 2$ días contra $3.4 \pm 2.2$ en la FD ( $p=0.004)$. La cefalea, dolor retroocular, artralgias, mialgias, tos, dolor faríngeo, congestión nasal y conjuntivitis fue similar en ambas formas de dengue. La diarrea y el vómito fueron más frecuentes en FHD $(p<0.05)$. En FD las manifestaciones hemorrágicas, fuga capilar y trombocitopenia ocurrieron en $24.8,6.7$ y $36.7 \%$, respectivamente; petequias, gingivorragia y epistaxis se asociaron a trombocitopenia menor de $20,000 / \mu \mathrm{L}(\mathrm{p}<0.05)$. Las demás manifestaciones hemorrágicas no se asociaron a trombocitopenia. Neutropenia, TTPA alargado, elevación de la TGO ocurrieron más en la FHD $(\mathrm{p}<0.05)$.

Conclusiones.- El género masculino, fiebre, vómito y gingivorragia se asocian fuertemente a FHD, pero no el haber padecido dengue; a mayor hemoconcentración, mayor severidad de la trombocitopenia.

\section{(Rev Biomed 2005; 16:87-94)}

Palabras clave: fiebre por dengue; fiebre hemorrágica por dengue; trombocitopenia; fuga capilar.

\section{SUMMARY.}

\section{Haemorrhagic manifestations in dengue.}

Objective.- Describe the clinical and epidemiological manifestations in patients with dengue fever (DF) and dengue haemorragic fever (DHF).

Materials and Methods.- A cross-sectional study of 231 cases of dengue taken care from the General Hospital of Culiacán "Dr. Bernardo J. Gastelum" from September to November, 2003, were classified according to the WHO criteria. The statistical program v6 Stata was used.

Results.- 209 (90.48\%) were DF and 22 (9.52\%) DHF. The mean age was $34.5 \pm 15$ years. The DF

Solicitud de sobretiros: Dr. José R. Rivas-Llamas, Departamento de Hematología del Hospital General de Culiacán "Dr. Bernardo J. Gastelum”, Juan Aldama y Nayarit S/N, Colonia, Rosales, C.P. 80230, Culiacán, Sinaloa, México.

Tel. (667) 71698 10, 71698 15, Ext. $151 \quad$ Correo electrónico: rivas@cln.megared.net.mx Recibido el 13/Septiembre/2004. Aceptado para publicación el 7/Enero/2005. 


\section{R Rivas-Llamas, RA Chaín-Castro, J Murillo-Llanes, MG Ramírez-Zepeda, T Gadea-Alvarez y col.}

predominated in women, with 129 cases $(61.7 \%)$, and DHF in men with 17 cases $(77.3 \%)$. For DHF patients went to the hospital in $4.7 \pm 2$ days, compared with $3.4 \pm 2.2$ for $\mathrm{DF}(\mathrm{p}=0.004)$. Headache, retroocular pain, arthralgias, diarrhea, and vomiting were more frequent in DHF $(\mathrm{p}<0.05)$. In DF the haemorrhagic manifestations, plasma leakage, and thrombocytopenia occurred in $24.8,6.7$, and $36.7 \%$ of cases respectively; petechias, gingivorragia, and epistaxis were associated with thrombocytopenia under $20,000 / \mu \mathrm{L}$ ( $\mathrm{p}<0.05$ ). Other haemorrhagic manifestations were not associated with thrombocytopenia. Neutropenia, lengthy APTT, elevation of the glutamicoxalacetic transaminase occurred more frequently in the DHF patients $(\mathrm{p}<0.05)$.

Conclusions.- The males with fever, vomiting, and gingivorragia are strongly associated with DHF, but not with having had dengue previously. Higher hemoconcentration is associated with the severity of thrombocytopenia. (Rev Biomed 2005; 16:87-94)

Key words: dengue fever; dengue hemorrhagic fever; thrombocytopenia; plasma leakage; Sinaloa México.

\section{INTRODUCCIÓN.}

El dengue es un síndrome febril agudo infeccioso, de origen viral, transmitido por los mosquitos Aedes aegypti y Aedes albopictus, siendo el primero el más eficaz y frecuente. Es la infección viral más común en el mundo transmitida por artrópodos, con una mayor incidencia en Asia, África, Centro y Sudamérica. El virus del dengue (VD) es un RNA virus del grupo de los flavivirus, de los cuales existen cuatro serotipos denominados Den-1, Den-2, Den-3 y Den-4. Deja inmunidad a largo plazo para variedades homotípicas y a corto plazo para las heterotípicas o de diferente serotipo (1).

El dengue tiene dos presentaciones clínicas: la fiebre por dengue (FD), también llamado dengue clásico y la fiebre hemorrágica por dengue (FHD), también llamado dengue hemorrágico. Las manifestaciones clínicas de ambos son diferentes, ya que la FD es una enfermedad autolimitada caracterizada por fiebre, mialgias y artralgias, cefalea, dolor ocular, rash, leucopenia y moderada trombocitopenia, cuyo curso clínico es de 5 a 7 días. En la FHD hay aumento en la permeabilidad capilar con extravasación vascular, manifestaciones hemorrágicas y daño hepático. En ambos casos las manifestaciones hemorrágicas son de diversa localización y grado de severidad. La pérdida de líquidos ocasionada por el daño endotelial puede ocasionar una importante depleción del volumen intravascular con el desarrollo del síndrome de choque por dengue ( $\mathrm{SChD}$ ). La participación de los sistemas hematológico, vascular y hepático, con la consecuente trombocitopenia, tendencia al sangrado, la fuga capilar y la elevación de las enzimas hepáticas, son los cambios más característicos en la FHD (2-4).

El mecanismo subyacente responsable del sangrado no tiene una explicación clara. La trombocitopenia es un hallazgo universal en la FHD y es uno de los criterios estipulados por la Organización Mundial de la Salud (OMS) para la definición del caso clínico, aunque también se ha encontrado en un 50\% de los casos de FD y se ha demostrado además disfunción plaquetaria e incremento en la actividad secretoria de las plaquetas (2).

Según datos de la OMS, cada año se presentan en el mundo 100 millones de casos nuevos de dengue, con 500,000 casos de FHD y 25,000 muertes. Los primeros casos de dengue en México se presentaron en 1941, con una posterior disminución por los programas de erradicación del mosco y una reaparición de la enfermedad en 1978, ingresando por Guatemala y difundiéndose al resto del país. De 1978 a 1999 se sumaron 418,281 casos de dengue, de los cuáles el 53\% correspondió a 7 estados: Veracruz, Tamaulipas, Guerrero, Sinaloa, Oaxaca, Nuevo León y Chiapas. En Sinaloa, durante 2002, se presentaron 4,691 casos de dengue, de los cuales el $12 \%$ fueron confirmados por laboratorio. En 2003 se diagnosticaron 7,201 casos, con el $12 \%$ de confirmados por laboratorio. En cuanto a la FHD, en 2002 se presentaron 75 casos contra 146 de 2003, con el $100 \%$ de confirmación por el laboratorio y hasta el momento se han obtenido nueve aislamientos virales: ocho son del tipo Den-2 y uno del Den-3 (Fuente:

\section{Revista Biomédica}


Manifestaciones hemorrágicas en el dengue.

Información de la Dirección General de Epidemiología de la SSA del Estado de Sinaloa, Noviembre de 2003).

Este trabajo se realizó con el propósito de hacer una descripción de las características clínicas y epidemiológicas en ambas formas de dengue, información que es útil para la detección temprana de estos casos y poder prevenir las complicaciones derivadas del propio padecimiento.

\section{MATERIAL Y MÉTODOS.}

Estudio transversal de 231 casos de dengue obtenidos mediante muestreo no aleatorio consecutivo que se atendieron en el Hospital General de Culiacán "Dr. Bernardo J. Gastelum" del primero de septiembre al 30 de noviembre de 2003. Fueron clasificados de acuerdo a la definición de caso de fiebre por dengue y fiebre hemorrágica por dengue de acuerdo a los criterios establecidos por la OMS/OPS (5), donde el caso de fiebre por dengue probable (FD) se caracteriza por cefalea, fiebre, dolor detrás de los ojos, artralgia, mialgia, rash, manifestaciones hemorrágicas, leucopenia. La fiebre hemorrágica por dengue (FHD) por cuadro clínico de fiebre por dengue, trombocitopenia menor de $100,000 / \mu \mathrm{L}$, manifestaciones hemorrágicas y fuga capilar (hemoconcentración, hipoalbuminemia, ascitis, derrame pleural o pericardico, liquido perivesicular). En ambos casos se consideró criterio epidemiológico para el diagnóstico de dengue, la presencia de casos de dengue confirmados en el mismo lugar y tiempo que el paciente en estudio y se excluyeron los casos que sufrieran cuadro clínico compatible con rikettsiosis, leptospirosis o hepatitis viral.

Se utilizó el cuestionario para el reporte de caso de dengue utilizado por la Dirección General de Vigilancia Epidemiológica de los Servicios de Salud de México, el cual incluyó las siguientes variables epidemiológicas: antecedente de dengue en la localidad, en la familia, cuadro previo de dengue, viajes a otras localidades en los 15 días previos al inicio de la sintomatología, edad, sexo, manifestaciones clínicas, exámenes de laboratorio como hemoglobina, hematocrito, leucocitos, neutrófilos, plaquetas, transaminasa glutámica oxalacética (TGO), transaminasa glutámica pirúvica (TGP), albúmina sérica, tiempo de protrombina (TP) y tiempo de tromboplastina parcial activada (TTPA), para lo cual se utilizó equipo automatizado CELL-DXN 3700 Abbott, Synchron CX5 CE Beckman y CA-500 Sysmex. La trombocitopenia fue considerada cuando la cuenta plaquetaria estuviera por debajo de 100,000 por $\mu \mathrm{L}$.

Para fines de análisis se formaron 6 grupos de trombocitopenia: grupo I: menos de 19 mil plaquetas; grupo II: de 20 a 39,000 plaquetas; grupo III de 40 a 59 mil plaquetas; grupo IV de 60 a 79 mil plaquetas; grupo V de 80 a 99 mil plaquetas y grupo VI más de 100 mil plaquetas. Se determinó su frecuencia y grado de asociación con las diferentes manifestaciones hemorrágicas.

Se determinaron medias, proporciones, razón de prevalencia, $\chi^{2}$ y la prueba exacta de Fisher para variables categóricas y regresión logística para variables categóricas para determinar el grado de asociación entre variables. Para variables cuantitativas se utilizó la prueba de T de Student y regresión lineal. Para determinar la significancia de los resultados se considero un error alfa de 0.05 , utilizándose el programa estadístico Stata versión 6.

Todos los pacientes firmaron carta de consentimiento informado.

\section{RESULTADOS.}

De 231 casos estudiados, 209 (90.48\%) presentaron fiebre por dengue, y $22(9.52 \%)$ fiebre hemorrágica por dengue, guardando una proporción de 9 a 1.

La FD fue más frecuente en la mujer con 129 casos $(61.72 \%)$, contrario a la FHD donde predominó el sexo masculino con $17(77.2 \%)(\mathrm{p}<0.05)$.

El tiempo transcurrido entre el inicio de los síntomas y la atención médica fue en general de 3.6 \pm 2.2 días. En los pacientes con FHD fue de $4.7 \pm 2.0$ días, contra $3.4 \pm 2.2$ días en la FD ( $p<0.05)$. Los signos y síntomas fueron similares en ambos grupos. Sin embargo, el vómito y la diarrea fueron más frecuentes en la FHD (cuadro 1). La distribución de los grupos de edad fue similar en ambas formas de 
R Rivas-Llamas, RA Chaín-Castro, J Murillo-Llanes, MG Ramírez-Zepeda, T Gadea-Alvarez y col.

\begin{tabular}{|c|c|c|c|}
\hline \multicolumn{4}{|c|}{$\begin{array}{c}\text { Cuadro } 1 \\
\text { Características clínicas de pacientes con fiebre por } \\
\text { dengue y fiebre hemorrágica por dengue }\end{array}$} \\
\hline $\begin{array}{l}\text { Variables } \\
\text { clínicas }\end{array}$ & $\begin{array}{c}\mathrm{FD} \\
\mathrm{n} / \mathrm{N}(\%)\end{array}$ & $\begin{array}{l}\text { FHD } \\
\mathrm{n} / \mathrm{N}(\%)\end{array}$ & ${ }^{*} \mathrm{p}$ \\
\hline Hombres & $80 / 209(38.2)$ & $17 / 22(77.27)$ & $<0.05$ \\
\hline Mujeres & $129 / 209(61.7)$ & $5 / 22(22.7)$ & $<0.05$ \\
\hline $\begin{array}{l}\text { Días } \\
\text { transcurridos }\end{array}$ & $\begin{array}{c}3.4 \pm 2.2 \\
n=194\end{array}$ & $\begin{array}{c}4.7 \pm 2.0 \\
n=22\end{array}$ & $<0.05$ \\
\hline Edad & $34.4 \pm 15.3$ & $34.3 \pm 130.94$ & \\
\hline Fiebre & $199 / 209(95.2)$ & $22 / 22(100 \%)$ & 0.29 \\
\hline Cefalea & 197/208(94.7) & $22 / 22(100)$ & 0.26 \\
\hline Dolor retrocular & 152/207(73.4) & $17 / 22(77.3)$ & 0.69 \\
\hline Artralgias & 190/209(90.9) & $21 / 22(95.4)$ & 0.47 \\
\hline Mialgias & 196/209(93.7) & $22 / 22(100)$ & 0.22 \\
\hline Rash & 40/208(19.2) & $5 / 22(22.7)$ & 0.69 \\
\hline Vómitos & $98 / 208(47.1)$ & 20/22(90.9) & $<0.05$ \\
\hline Diarrea & 45/208(21.6) & 9/22(40.9) & $<0.05$ \\
\hline Tos & 41/208(19.7) & $2 / 22(18.1)$ & 0.80 \\
\hline Dolor abdominal & $53 / 209(25.3)$ & $9 / 22(41) 0.11$ & \\
\hline
\end{tabular}

*datos analizados mediante el programa estadístico Stata v6. $\mathrm{FD}=$ fiebre por dengue; $\mathrm{FHD}=$ fiebre hemorrágica por dengue; $\mathrm{n}=$ número de casos; $\mathrm{N}=$ población estudiada.

dengue, aunque predominó el grupo de 25 a 44 años de edad en los pacientes con FHD (cuadro 2).

Las manifestaciones hemorrágicas ocurrieron sólo en 74 pacientes (32.0\%); éstas ocurrieron en todos los pacientes con FHD y en 52 (24.8\%) casos de pacientes con FD. Las manifestaciones más comunes en ambos grupos fueron las petequias, gingivorragia, y epistaxis. Sin embargo fueron más frecuentes y más severas en la FHD (cuadros 3 y 4).

Entre los antecedentes epidemiológicos analizados, como procedencia urbana de los casos, el antecedente de dengue en la localidad, cuadro similar en la familia, dengue previo y viaje en los 15 días previos al inicio del padecimiento no hubo diferencias en los grupos.

El hematocrito fue de $41.5 \pm 5.0 \%$ versus 46.8 $\pm 6.4 \%$, la hemoglobina $14.2 \pm 1.6 \mathrm{~g} / \mathrm{dL}$ versus $16 \pm$ $2.2 \mathrm{~g} / \mathrm{dL}$, leucocitos de $4746 \pm 2003 \mu \mathrm{L}$ versus Revista Biomédica
$4466.471 \pm 1751 \mu \mathrm{L}$ y neutrofilos $2887 \pm 1736 \mu \mathrm{L}$ versus $2003 \pm 949 \mu \mathrm{L}$, plaquetas $141,283 \pm 93,339$ $\mu \mathrm{L}$ versus $27,732 \pm 22,015 \mu \mathrm{L}$, en los pacientes con FD y FHD, respectivamente. Estos valores fueron diferentes estadísticamente, excepto para los leucocitos.

La TGO de $81.1 \pm 107 \mathrm{UI} / \mathrm{dL}$ versus $137.5 \pm$ $140 \mathrm{UI} / \mathrm{dL}$ y la TGP fue de $66.6 \pm 81 \mathrm{UI} / \mathrm{dL}$ versus $82.3 \pm 82 \mathrm{UI} / \mathrm{dL}$, en los pacientes con FD y FHD respectivamente. Las diferencias sólo fueron significativas para la TGO ( $\mathrm{p}<0.05)$. El TP fue similar en ambas variedades de dengue, y el TTPA fue de $37.2 \pm 5.7 \mathrm{~s}$ versus $41.9 \pm 5.8 \mathrm{~s}$ en los casos con FD y FHD, respectivamente $(\mathrm{p}<0.05)$.

La hipoalbuminemia fue la manifestación de fuga capilar más frecuente en ambos casos de dengue, con 24 casos, seguido por hemoconcentración con 16 , ascitis con 14 casos, derrame pleural en 9 y derrame pericárdico en un sólo caso (cuadro 5).

\section{DISCUSIÓN.}

El dengue, y sobre todo la FHD y el SCHD pueden convertirse en verdaderas urgencias epidemiológicas, cuya magnitud puede rebasar la capacidad de respuesta de los servicios médicos asistenciales. Incrementa la mortalidad en los extremos de la vida (1-3), además de afectar principalmente a los grupos de edad más productivos (5), situación que

\section{Cuadro 2}

Frecuencia de los grupos de edad de acuerdo a la forma de dengue.

\begin{tabular}{llll}
\hline Grupos de edad & $\begin{array}{c}\text { FD } \\
\mathrm{n}(\%)\end{array}$ & $\begin{array}{c}\text { FHD } \\
\mathrm{n}(\%)\end{array}$ & *p \\
\hline$<$ 14 años & $3 / 205(1.46)$ & $0 / 22(0 \%)$ & $>0.05$ \\
15-24 años & $62 / 205(30.24)$ & $6 / 22(27.27)$ & $>0.05$ \\
25-44 años & $91 / 205(44.39)$ & $12 / 22(54.55)$ & $>0.05$ \\
45-64 años & $41 / 205(20.00)$ & $3 / 22(13.64)$ & $>0.05$ \\
Más de 64 años & $8 / 205(3.90)$ & $1 / 22(4.55)$ & $>0.05$ \\
\hline
\end{tabular}

*Análisis univariado de los grupos de edad mediante la $\chi^{2}$ del programa Stata versión 6; FD= fiebre por dengue; $\mathrm{FHD}=$ fiebre hemorrágica por dengue 


\section{Manifestaciones hemorrágicas en el dengue.}

\section{Cuadro 3}

Frecuencia de las manifestaciones hemorrágicas entre los pacientes con dengue clásico y la fiebre hemorrágica por dengue.

\begin{tabular}{lccc}
\hline $\begin{array}{l}\text { Variables } \\
\text { hemorrágicas }\end{array}$ & $\begin{array}{c}\mathrm{FD} \\
\mathrm{n} / \mathrm{N}(\%)\end{array}$ & $\begin{array}{c}\mathrm{FHD} \\
\mathrm{n} / \mathrm{N}(\%)\end{array}$ & ${ }^{*} \mathrm{P}$ \\
\hline Petequia & $15 / 209(7.18)$ & $5 / 22(22.73)$ & 0.014 \\
Equimosis & $2 / 209(0.96)$ & $1 / 22(4.55)$ & 0.157 \\
Epistaxis & $9 / 209(4.31)$ & $6 / 22(27.27)$ & $<0.05$ \\
Gingivorragia & $12 / 209(5.74)$ & $11 / 22(50.0)$ & $<0.05$ \\
Hematemesis & $7 / 208(3.37)$ & $4 / 22(18.18)$ & $<0.05$ \\
Melena & $1 / 209(0.48)$ & $5 / 21(23.81)$ & $<0.05$ \\
Hematuria & $6 / 208(2.88)$ & $3 / 21(14.29)$ & $<0.05$ \\
Torniquete & $13 / 206(6.31)$ & $5 / 22(22.7)$ & $<0.05$ \\
\hline
\end{tabular}

${ }^{*}$ Datos analizados mediante la aplicación de la $\chi^{2}$ y la prueba exacta de Fisher o el programa estadístico Stata versión 6. FD: fiebre por dengue; FHD: fiebre hemorrágica por dengue; $\mathrm{n}=$ número de casos; $\mathrm{N}=$ población estudiada encontramos en nuestra serie (cuadro 2).

Las manifestaciones clínicas de nuestra serie son semejantes a lo descrito en otras publicaciones (1,2, 4). Es importante destacar que la presencia de vómito persistente y diarrea fueron más frecuentes en los pacientes con FHD, lo que debe alertar a la posibilidad de una forma grave de la enfermedad.

A pesar de que algunos pacientes presentaron trombocitopenia severa, ninguno presentó sangrado grave. Las manifestaciones hemorrágicas más frecuentes en la FHD fueron gingivorragia, epistaxis y las petequias. Sólo un paciente presentó signos inminentes de síndrome de choque por dengue (SHCD), que se resolvió satisfactoriamente en 24 horas con la reposición de líquidos.

La neutropenia, lo mismo que la elevación de la TGO, hipoalbuminemia y prolongación del TTPA, fueron más acentuados en la FHD. Esto está acorde con lo ya informado por otros autores y se ha relacionado con una mayor gravedad de la enfermedad (1-4), en relación a los profundos cambios fisiológicos e inmunológicos que se observan en el

Cuadro 4

Asociación entre los grupos de trombocitopenia y las variables hemorrágicas*.

\begin{tabular}{lcccccc}
\hline $\begin{array}{l}\text { Variables } \\
\text { hemorrágicas }\end{array}$ & $\begin{array}{c}\text { Grupo I } \\
\text { OR / IC95\% }\end{array}$ & $\begin{array}{c}\text { Grupo II } \\
\text { OR / IC95\% }\end{array}$ & $\begin{array}{c}\text { Grupo III } \\
\text { OR / IC95\% }\end{array}$ & $\begin{array}{c}\text { Grupo IV } \\
\text { OR / IC95\% }\end{array}$ & $\begin{array}{c}\text { Grupo V } \\
\text { OR / IC95\% }\end{array}$ & $\begin{array}{c}\text { Grupo VI } \\
\text { OR / IC95\% }\end{array}$ \\
\hline Epistaxis & 4.5 & -- & -- & 1.3 & -- & -- \\
& $(1.4-14)$ & & & $(0.16-11.5)$ & & \\
Petequia & 3.1 & 3.9 & -- & -- & 1.05 & -- \\
& $(1.0-9.8)$ & $(1.3-11.0)$ & & & $(0.12-8.6)$ & \\
Gingivorragia & 3.4 & 2.3 & 2.5 & 2.0 & 2.0 & -- \\
& $(1.22-9.9)$ & $(0.78-6.9)$ & $(0.75-8.2)$ & $(0.4-10.2)$ & $(0.41-10.2)$ & -- \\
Hematemesis & 2.0 & -- & 2.6 & 2.1 & -- & - \\
& $(0.4-9.9)$ & & $(0.51-13.3)$ & $(0.25-19.0)$ & & - \\
Melena & 8.5 & -- & 2 & 4 & -- & 1.2 \\
& $(1.6-45.1)$ & & $(0.42-18.2)$ & $(0.42-37.7)$ & & $(0.14-10.2)$ \\
\hline
\end{tabular}

* Los datos fueron analizados mediante regresión logística con el programa Stata versión 6.

†Grupo I: < 19,000; grupo II: 20 a 39 mil; grupo III: 40 a 59 mil; grupo IV: 60 a 79 mil; grupo V: 80 a 99 mil plaquetas por mm; grupo VI: más de 100 mil plaquetas.łrazón de momios obtenida mediante regresión logística bivariada. 
R Rivas-Llamas, RA Chaín-Castro, J Murillo-Llanes, MG Ramírez-Zepeda, T Gadea-Alvarez y col.

Cuadro 5

Frecuencia de manifestaciones de fuga capilar por tipo de dengue.

\begin{tabular}{llll}
\hline & \multicolumn{1}{c}{ FD } & FHD & Total \\
$\mathrm{n} / \mathrm{N}(\%)$ & $\mathrm{n} / \mathrm{N}(\%)$ & $14 / 231(6.0)$ \\
& $5 / 209(2.39)$ & $9 / 22(40.9)$ & $10 / 231(4.3)$ \\
Ascitis & $6 / 209(2.87)$ & $4 / 22(18.18)$ & $1 / 209(0.43)$ \\
Derrame pleural & $0 / 209(0)$ & $1 / 22(4.5)$ & $24 / 57(42.1)$ \\
Derrame pericárdico & $16 / 16(100)$ & $16 / 216(7.4)$ \\
Hipoalbuminemia & $8 / 41(19.5)$ & $7 / 22(31.8)$ & \\
Hemoconcentración & $9 / 194(4.6)$ & & \\
\hline
\end{tabular}

$\mathrm{FD}=$ fiebre por dengue; $\mathrm{FHD}=$ fiebre hemorrágica por dengue; $\mathrm{n}=$ número de casos; $\mathrm{N}=$ población estudiada.

dengue.

Se encontró asociación entre la trombocitopenia menor de $20,000 / \mu \mathrm{L}$ y las manifestaciones de sangrado, contrario a lo señalado por otros autores (7).

El sangrado en la FHD no tiene aún una explicación satisfactoria. Se ha mencionado que existe un aumento en la actividad procoagulante $\mathrm{y}$ fibrinolítica, así como la disminución de los anticoagulantes naturales, sobre todo las proteínas $\mathrm{C}$ y $\mathrm{S}$ y la antitrombina III, lo que haría que se desencadenaran cambios semejantes a los observados en la coagulación intravascular diseminada (CID), con complicaciones trombóticas y manifestaciones de sangrado. Sin embargo en el dengue esto no ocurre o son mínimos (2). La disminución de los anticoagulantes naturales se ha explicado por la disminución de su síntesis en el hígado, o por fuga capilar, ya que los pesos moleculares de estas tres proteínas varían de 30 a 70,000 daltons, semejantes al de la albúmina, que también sufre este fenómeno (2). Todo ello hace pensar que el daño hepático es importante y sitúa al hepatocito, junto con el monocito y la célula endotelial, como las más afectadas en la enfermedad $(1,4)$.

La infección secundaria, debido a la presencia de anticuerpos heterotípicos provocados por una infección previa por otro serotipo, facilita la entrada del nuevo virus al monocito, ya que los anticuerpos circulantes no son capaces de neutralizar a este nuevo serotipo. La gran replicación viral generada aumenta la carga viral de la infección haciéndola más severa. Al lisarse el monocito se liberan citocinas (IL-1, IL-
2, IL-6, TNF e interferones alfa y gamma) que desencadenan el proceso inmunopatológico de la enfermedad. Se provoca una mayor producción de linfocitos CD8 que CD4, lo que disminuye la posibilidad del sistema inmune para combatir la infección $(4,8)$.

La liberación de las citocinas lesiona directamente al monocito, la célula endotelial y el hepatocito, aumentando el daño vascular, la permeabilidad vascular y la fuga capilar, que aunado al aumento en la producción de trombomodulina, crean una alteración en el balance de las actividades procoagulante y anticoagulante, incrementando la tendencia a la hemorragia (8). La formación de complejos inmunes activan al complemento $\mathrm{C} 3$ a y $\mathrm{C} 5 \mathrm{a}$, que en presencia de IFN-gamma y TNF aumentan la lesión endotelial $(3,4)$ y destruyen plaquetas, siendo éste uno de los mecanismos de trombocitopenia (9), la que también se ha explicado por fuga capilar, adhesión al endotelio dañado, disminución en su producción a nivel medular y disminución en su supervivencia $(4,10,11)$.

La activación de la fibrinólisis es debida a la mayor actividad de la plasmina por aumento en la liberación del factor activador del plasminógeno (tPA). El antagosnista de factor es el inhibidor del activador del plasminógeno (PAI-1), que es producido por las plaquetas, el hepatocito y el endotelio. Durante la actividad de la FHD el índice tPA/PAI-1 está muy aumentado. El aumento de este índice, así como la prolongación del TTPA, correlacionan con la severidad de la enfermedad y pudieran ser marcadores

\section{Revista Biomédica}




\section{Manifestaciones hemorrágicas en el dengue.}

tempranos de FHD y SCHD. Durante la convalecencia y la recuperación del paciente con FHD aumentan los niveles de PAI-1 y de plaquetas, disminuyendo los niveles de tPA y recuperándose el TTPA, lo que está de acuerdo con la mejoría del paciente (4).

La sobreproducción de IL-6 juega un papel muy importante en el aumento en la producción de anticuerpos antiplaquetarios, la elevación de los niveles de tPA y la deficiencia de Factor XII de la coagulación en la vía intrínseca, aumentando la tendencia hemorrágica. La prolongación del TTPA se debe a un aumento en el consumo y a una disminución en la producción de los factores de la coagulación por daño hepático, mismos que explican también la elevación de las transaminasas (4), como observamos en nuestro trabajo.

Todo demuestra que el daño a la célula endotelial es debido al efecto citopático directo del virus y al daño inmune mediado por el reclutamiento de leucocitos y los anticuerpos antidengue. Y dado que el endotelio juega un papel crucial en el mantenimiento de la homeostasis, el daño en la célula endotelial por la infección del virus del dengue cambia el balance entre la procoagulación y anticoagulación del endotelio y aumenta la tendencia al sangrado $(4,12)$.

Aunque no existe una relación de causa a efecto, sí hay una asociación entre la severidad de la enfermedad, el grado de trombocitopenia, la prolongación del TTPA, el aumento en los niveles de hemoglobina y hematocrito y la disminución en la cuenta de neutrófilos. La alteración de estas variables tiene una explicación patogénica, cada una por separado y una no es causa de la otra, como ya se comentó anteriormente.

Debido a que al inicio son muy difíciles de diferenciar las manifestaciones clínicas de las dos variedades de dengue y el hecho de no contar con marcadores tempranos, la prolongación del TTPA podría tomarse como un marcador temprano de la FHD, lo que coincide con los datos clínicos de disminución de la fiebre y las manifestaciones clínicas de fuga capilar.
La transfusión de concentrados plaquetarios dependerá de la condición clínica del paciente, la cuenta de plaquetas y las manifestaciones de sangrado que presente, siendo el manejo simultáneo con reposición de líquidos y electrolitos, aporte de factores de la coagulación y transfusión de PG cuando el caso lo requiera. En nuestra serie sólo usamos concentrados plaquetarios en tres casos, uno de ellos con sangrado digestivo grave por úlcera gástrica y los otros dos con sangrado activo a diferentes niveles.

\section{REFERENCIAS.}

1.- Kautner I, Robinson MJ, Kuhnle U. Dengue and dengue hemorrhagic fever. J Pediatr 1997; 131: 516-24.

2.- Wills BA, Oragui EE, Stephens AC, Daramola OA, Dung NM, Loan HT, et al. Coagulation abnormalities in dengue hemorrhagic fever: serial investigations in 167 vietnamese children with dengue shock syndrome. Clin Infect Dis 2002; 35: 277-85.

3.- Gubler, DJ. Dengue and dengue hemorrhagic fever. Clin Microbiol Rev 1998; 11: 480-96.

4.- Lei HY, Yeh TM, Liu HS, Lin YS, Chen SH, Liu CC. Immunopathogenesis of dengue virus infection. J Biomed Sci 2001; 8: 377-88.

5.- Organización Panamericana de la Salud. Boletín Epidemiológico. Normas y Estándares en Epidemiología. Definiciones de casos: Dengue y Leptospirosis. 2000, Jun; Vol $21 \mathrm{~N}^{\circ} 2$.

6.- Rodríguez-Arias O, Pérez-Péres A, Despaigne-Bicet A, Irarragirri-Dorado CA. Caracterización de pacientes con diagnóstico presuntivo de dengue en el brote epidémico del año 1997. Rev Cub Med Trop 2001; 53: 24-7.

7.- Lum LC, Goh AY, Keong PW, El-Amin AM, Lam SK. Risk factors for hemorrhage in severe dengue infections. J Pediatr 2002; 140:629-31.

8.- Juffrie M, van der Meer GM, Hack CE, Haasnoot K, Sutaryo AJ, Veerman AJP, Thijs LG. Inflamatory mediators in dengue virus infection in children: IL-6 and its relation to $\mathrm{C}$ reactive protein and secretory phospholipase A2. Am J Trop Med Hyg 2001; 65: 70-5. 
R Rivas-Llamas, RA Chaín-Castro, J Murillo-Llanes, MG Ramírez-Zepeda, T Gadea-Alvarez y col.

9.- Oishi K, Inoue S, Cinco MT, Dimaano EM, Alera MT, Alfon JAR, et al. Correlation between increased plateletassociated $\operatorname{IgG}$ and thrombocytopenia in secondary dengue virus infection. J Med Virol 2003; 71: 259-64.

10.- Krishnamurti C, Peat RA, Cutting MA, Rothwell SW. Platelet adhesion to dengue-2 virus infected endothelial cells. Am J Trop Med Hyg 2002; 66:435-41.

11.- Isarangkura P, Tuchinda S. The behavior of transfused platelets in dengue hemorrhagic fever. Sotheast Asian J Trop Med Publ Health 1993; 24 (Suppl 1): 222-4.

12.- Levi M, Cate HT, van der Poll T. Endothelium: interface between coagulation and inflammation. Crit Care Med 2002; 30 (Suppl 5): S220-4.

\section{Revista Biomédica}

